\title{
Eficacia disolvente y citotoxicidad del aceite de cáscara de limón (Citrus limon)
}

\author{
Solvent efficiency and cytotoxicity of lemon peel oil (Citrus limon) \\ Paul Herrera-Plasencia ${ }^{1, a, b}$, Carmen Garcia-Rupaya ${ }^{2, b}$, Leyla Delgado-Cotrina ${ }^{2, b}$

\section{RESUMEN}

Objetivo: Evaluar in vitro el efecto disolvente y tóxico del aceite de cáscara de limón (Citrus limon) según tiempo de exposición e identificar sus componentes a través de la cromatografía de gases. Material y métodos: El estudio fue de tipo experimental in vitro. Para determinar el efecto disolvente se utilizaron conos de gutapercha estandarizados de endodoncia número 80 con conicidad 0,02; los disolventes utilizados fueron: xilol, aceite esencial de cáscara de limón y naranja; éstos fueron sumergidos en 2, 5 y 10 minutos $(n=8)$. Se registró el peso antes y después de la inmersión a las sustancias evaluadas para determinar la pérdida de masa. Para evaluar la toxicidad se emplearon Artemias franciscanas a través de la concentración letal media; la cromatografía de gases se utilizó para obtener los componentes de cada aceite. Para comparar el efecto disolvente entre las sustancias se empleó el análisis ANOVA/Tukey. Resultados: No se encontró diferencia significativa para disolver la gutapercha con el aceite esencial de cáscara de limón, de naranja y xilol ( $\mathrm{p}>0.05)$. El tiempo no tuvo influencia para la disolución de la gutapercha ( $\mathrm{p}>0.05)$. Todos los aceites fueron tóxicos, a excepción del aceite de limón 25\%. Conclusiones: El Limoneno y el D limoneno fueron los componentes con mayor porcentaje en el aceite de limón y de naranja, respectivamente. El aceite de limón podría ser una considerada como alternativa para ser utilizado como disolvente de gutapercha de uso endodóntico, por tener efecto similar que el aceite naranja.

PALABRAS CLAVE: Gutapercha; solventes; aceites volátiles; toxicidad. 


\section{SUMMARY}

Objective: To evaluate in vitro the solvent and toxic effect of lemon peel oil (Citrus limon) according to exposure time. Its components were identified through gas chromatography. Material and Methods: The study was of experimental type in vitro. To determine the solvent effect, standardized gutta-percha cones of endodontic number 80 with taper 0.02 were used; solvents used were: xylol, lemon and orange peel essential oil; these were immersed in 2, 5 and 10 minutes $(\mathrm{n}=8)$. The weight was recorded before and after the immersion to the substances evaluated to determine the loss of mass. To evaluate the toxicity, Artemias Franciscan were used through the medium lethal concentration; Gas chromatography was used to obtain the components of each oil. The ANOVA / Tukey analysis was used to compare the solvent effect between the substances. Results: No significant difference was found to dissolve the gutta-percha with the lemon, orange and xylol essential oil ( $p>$ 0.05). Time did not influence the dissolution of gutta-percha ( $>>0.05)$. All oils were toxic, with the exception of $25 \%$ lemon oil. Conclusions: Limonene and D limonene were the components with the highest percentage in lemon and orange oil, respectively. Lemon oil could be considered as an alternative to be used as a gutta-percha solvent for endodontic use, because it has a similar effect as orange oil.

\section{KEYWORDS: Guttapercha, solvents; volatiles oils; toxicity.}

\section{INTRODUCCIÓN}

El retratamiento endodóntico tiene como objetivo remover los materiales del conducto radicular, verificar las deficiencias o reparar los defectos de origen patológico o iatrogénico (1). Los métodos para retirar el material de obturación pueden ser térmicos, mecánicos, químicos o la combinación de ellos $(1,2)$. El método químico asociado con el mecánico es el más utilizado hoy en día. Los solventes reblandecen la gutapercha facilitando el acceso de los instrumentos al foramen apical para así remover todo el material obturador; se han recomendado disolventes tales como cloroformo, metilcloroformo, eucaliptol, halotano, aguarras modificado y xileno, todos con cierto nivel de toxicidad (3). Como opción a estos solventes se están utilizando algunos de origen natural, como el eucaliptol y naranja, debido a sus capacidades de disolver la gutapercha, siendo en la actualidad alternativas debido a su menor toxicidad. Los aceites esenciales de los cítricos son normalmente obtenidos de sus cáscaras; son una mezcla de sustancias volátiles, lipofílicas, generalmente odoríferas, líquidas, incoloras y acarameladas. Los aceites esenciales obtenidos de la cáscara de naranja o del limón son usados para dar sabor a las bebidas y otros alimentos, para la fabricación de medicamentos, cosméticos y perfumes (4).

En odontología, Pécora et al.,propusieron la utilización del aceite de cáscara de naranja para desintegrar el cemento de óxido de zinc-eugenol (5). Pécora et al., observaron que el cloroformo reblandeció más rápidamente la gutapercha, seguido del xilol y aceite de cáscara naranja (6). Magalhaes et al., encontraron que el cloroformo, el aceite esencial de cáscara de naranja y eucaliptol presentaron similar capacidad de solvencia (7). Pineda et al., encontraron que el aceite de naranja y eucaliptol presentaron efectos solventes similares (8). Es importante conocer que entre los 200 componentes hay dos importantes, el limoneno que representa la fracción terpénica, y el linalol, representante de la fracción oxigenada (9).

Por otro lado, la citotoxicidad de los solventes debe ser considerada, como los estudios realizados por Nagayama y Zaccaro et al., quienes usaron líneas celulares, concluyeron que el aceite de naranja, eucaliptol, xilol, cloroformo y halotano son citotóxicos, siendo el aceite de naranja menos citotóxico por permitir alguna viabilidad celular $(10,11)$; el alto costo de estos experimentos, así como el sufrimiento que se causa a los animales ha hecho que se reduzcan estas pruebas.

El ensayo de letalidad de Artemia, evalúa la toxicidad utilizando larvas de este crustáceo reproducidas en el laboratorio. Esta metodología fue propuesta por Michael y et al. (1956) ${ }^{13}$ con el motivo de tener como alternativa una herramienta útil y sencilla para evaluar la toxicidad, a través de la concentración letal media (CL50) (12). Se utiliza para identificar toxinas de hongos, toxicidad de extractos de 
plantas o predecir citoxicidad (14-17). El método de evaluación de citotoxicidad con artemias es una herramienta útil para predecir la toxicidad aguda por vía oral en extractos de plantas (18) o nanopartículas en biomedicina. Las artemias franciscanas presentan una baja variabilidad genética, ciclo de vida corto y las pruebas de citotoxicidad son de bajo costo (19).

El objetivo de la presente investigación fue evaluar la acción solvente y tóxica de un producto a base de aceite cáscara del limón (Citrus limón) sobre la gutapercha endodóntica y determinar sus componentes a través de cromatografía de gases.

\section{MATERIAL Y MÉTODOS}

La presente investigación se envió al Comité Institucional de Ética (CIE) de la Universidad Peruana Cayetano Heredia, siendo categorizado como exonerado de revisión por ser un estudio in vitro, con código 60681. Se evaluó la capacidad solvente del aceite de cáscara de naranja Citrus sinesis (Maquira, Maringá, Brasil), aceite de cáscara de limón Citrus limón (Esquisa, Lima, Perú) en las concentraciones del $100 \%, 75 \%, 50 \%$ y $25 \%$; el xilol (Xilodent, Proquident, Colombia) para el control positivo, y agua destilada (Martinotti, Lima, Perú) como control negativo.

Para esta investigación, se utilizaron conos de gutapercha estandarizados $\mathrm{N}^{\circ} 80$ de conicidad 0,02 (Dentsply, Maillefer, Suiza) lote $\mathrm{N}^{\mathrm{o}} 020610$ y fueron seleccionados aleatoriamente $(\mathrm{n}=8)(7)$. Los tiempos evaluados fueron de 2, 5 y 10 min para cada solvente (xilol, aceite cáscara de naranja, aceite cáscara de limón al 100\%, 75\%, 50\% y 25\% y agua destilada). Para determinar la capacidad solvente se empleó la diferencia de peso luego de sumergir los conos en las sustancias $(7,8)$; para esto se determinó el peso de los conos antes y después de la inmersión a los solventes en una balanza digital FA2104N (Jinghai Zhengji Instruments, Shanghai, China). Para la inmersión se utilizó un vial de color ámbar que contenía $5 \mathrm{ml}$ de cada solvente, la parte más ancha del cono fue sumergida en el solvente dejando $4 \mathrm{~mm}$ del extremo más delgado dirigido hacia arriba que quedó sin sumergir en todos los conos para ser sujetados con un alambre de ortodoncia $\mathrm{N}^{\circ} 16$ que envolvía el extremo no expuesto. Una vez transcurrido el tiempo indicado, se retiró el cono de gutapercha del solvente, el cual fue 1levado a un desecador ODHG-9053A (Jiangsu Zhengji Instruments, Shanghai, China) por $24 \mathrm{~h}$ en $37 \pm 1^{\circ} \mathrm{C}$. Posteriormente, se determinó el peso final. Para determinar la pérdida de peso se restó el peso final menos el peso inicial.

Para el ensayo de toxicidad se utilizaron una muestra de 15 a 20 nauplios (12). Cada ensayo se realizó por triplicado y se preparó $1 \mathrm{~L}$ de solución de sal marina al 3,8 \% en agua destilada y se incubó en un frasco de vidrio estéril transparente rotulando la fecha, la cantidad de solución salina y la hora en que los huevos de Artemia franciscana se añadieron a la solución. En este recipiente se agregó $30 \mathrm{~mL}$ de solución salina y $0.1 \mathrm{~g}$ de huevos de Artemia franciscana, se cubrió la parte superior del frasco con papel de aluminio y se dejó que la mezcla se hidrate por $1 \mathrm{~h}$. Luego se añadió 370 $\mathrm{mL}$ de solución salina al frasco. Se esperó $26 \mathrm{~h}$ para que la mayoría de los huevos de Artemia franciscana eclosionen, se anotó el momento en que se comenzó y terminó la colocación de los nauplios de Artemia franciscana en los tubos de ensayo con los solventes los que permanecieron $24 \mathrm{~h}$ cerca de una fuente de luz. Transcurrido este tiempo se realizó el conteo de los nauplios de Artemias franciscanas vivas (presencia de movilidad) y muertas (sin movilidad) y los nauplios totales. Cada solvente se evaluó por triplicado.

Para determinar los compuestos del aceite de cáscara de limón $100 \%$ se utilizó el cromatógrafo de gases (Agilent, California, USA), en el laboratorio de productos naturales de la Universidad Peruana de Cayetano Heredia. Para el análisis de los datos se usó la prueba de ANOVA/Tukey. La concentración letal media $\left(\mathrm{CL}_{50}\right)$ se analizó para cada serie individual mediante el análisis Probit que evalúa el grado de toxicidad del extracto de acuerdo con las siguientes categorías: extremadamente tóxico $\left(\mathrm{CL}_{50}<10 \mathrm{ug} / \mathrm{ml}\right)$, muy tóxico $\left(10<\mathrm{CL}_{50}<100 \mathrm{ug} / \mathrm{ml}\right)$, moderadamente tóxico $\left(100<\mathrm{CL}_{50}<1000 \mathrm{ug} / \mathrm{ml}\right)$

\section{RESULTADOS}

Los valores de la pérdida de peso de los conos de gutapercha luego de la exposición a los diferentes solventes se muestran en la tabla 1. La mayor pérdida de peso a los $2 \mathrm{~min}, 5 \mathrm{~min}$ y $10 \mathrm{~min}$ fue luego de la exposición al aceite esencial de cáscara de naranja 
Tabla 1. Promedios de las diferencias de pesos de la gutapercha en gramos en las sustancias y tiempos evaluados

\begin{tabular}{lcccccc}
\hline \multicolumn{1}{c}{ Sustancia } & \multicolumn{2}{c}{$\mathbf{2 ~ m i n}$} & \multicolumn{2}{c}{$\mathbf{5 ~ m i n}$} & \multicolumn{2}{c}{$\mathbf{1 0}$ min } \\
& Promedio & DS & Promedio & DS & Promedio & DS \\
\hline AECL 100\% & 0,003 & 0,002 & 0,002 & 0,001 & 0,003 & 0,001 \\
AECN & 0,004 & 0,002 & 0,004 & 0,000 & 0,004 & 0,001 \\
Xilol & 0,001 & 0,001 & 0,001 & 0,002 & $-0,002$ & 0,001 \\
Agua Destilada & 0,000 & 0,000 & 0,000 & 0,001 & 0,000 & 0,000 \\
AECL 75\% & 0,003 & 0,002 & 0,002 & 0,001 & 0,003 & 0,001 \\
AECL 50\% & 0,000 & 0,000 & 0,000 & 0,000 & 0,001 & 0,000 \\
AECL 25\% & 0,000 & 0,001 & 0,001 & 0,000 & 0,000 & 0,000 \\
\hline
\end{tabular}

Nota: los valores están expresados en $\mathrm{g}$.

AECL : Aceite esencial cáscara de limón

AECN : Aceite esencial cáscara de naranja

Tabla 2. Nivel de significancia de las comparaciones de las diferencias de los pesos de la gutapercha en las sustancias evaluadas

\begin{tabular}{lcccccc}
\hline \multicolumn{1}{c}{ Sustancias } & AECL 25\% & AECL 50\% & AECL 75\% & Agua Destilada & Xilol & AECN \\
\hline AECL 100\% & 0,001 & 0,001 & 0,260 & 0,001 & 0,440 & 0,635 \\
AECN & 0,003 & 0,008 & 0,721 & 0,008 & 1,000 & \\
xilol & 0,008 & 0,021 & 0,875 & 0,021 & & \\
Agua Destilada & 1,000 & 1,000 & 0,392 & & & \\
AECL 75\% & 0,229 & 0,395 & & & & \\
AECL 50\% & 1,000 & & & & & \\
\hline
\end{tabular}

Prueba de análisis de Tukey; $\mathrm{p}>0.05$

AECL : Aceite esencial cáscara de limón

AECN : Aceite esencial cáscara de naranja

Tabla 3. Valores de $\mathrm{LC}_{50}$ de las sustancias evaluadas empleando el ensayo de letalidad con Artemia franciscana.

\begin{tabular}{ccc}
\hline Sustancia & LC $_{\mathbf{5 0}} \mathbf{u g} / \mathbf{m L}$ & Intervalos de confianza al 95\% \\
\hline Xilol & 258 & $245,6-266,6$ \\
AECL 100\% & 38 & $31,72-46,04$ \\
AECL 75\% & 35 & $21,86-30,60$ \\
AECL 50\% & 34 & $13,71-26,45$ \\
AECL 25\% & $*$ & $*$ \\
AECN & 30 & $22,34-36,51$ \\
\hline
\end{tabular}

Análisis Probit (Finney 1971), del ensayo de letalidad realizado por triplicado.

AECL: Aceite esencial cáscara de limón

AECN: Aceite esencial cáscara de naranja

$\mathrm{LC}_{50}$ : Concentración letal media

* : No se determinó el LC50 en el 25\% porque todos los nauplios vivieron

$(0,004$ gr) y la menor pérdida de peso se obtuvo con el agua destilada para todos los tiempos, el aceite de cáscara de limón al 50 y $25 \%$ a los 2 min, aceite de cáscara de limón $50 \%$ a los 5 min y xilol a los 10 min. No se encontró diferencia significativa entre en la pérdida de peso de los conos de gutapercha expuestos a aceite de cáscara limón $100 \%$, aceite de limón $75 \%$, aceite de cáscara de naranja y xilol a los 2,5 y $10 \min (p>0,05)$. En la tabla 2 se muestra la concentración letal media $\left(\mathrm{LC}_{50}\right)$ de las sustancias evaluadas encontrando que el xilol presentó la $\mathrm{CL}_{50}$ de $258 \mathrm{ug} / \mathrm{mL}$, encontrándose en el grado de 
moderadamente tóxico $\left(100<\mathrm{CL}_{50}<1000 \mathrm{ug} / \mathrm{ml}\right)$; el aceite de cáscara de limón $100 \%$ presentó una $\mathrm{CL}_{50}$ de $38 \mathrm{ug} / \mathrm{ml}$, el aceite de limón $75 \%$ una $\mathrm{CL}_{50}$ de $35 \mathrm{ug} / \mathrm{ml}$, el aceite de limón $50 \%$ una $\mathrm{CL}_{50}$ de $34 \mathrm{ug} / \mathrm{ml}$ y el aceite de cáscara de naranja $\mathrm{CL}_{50}$ de $30 \mathrm{ug} / \mathrm{ml}$, encontrándose estos últimos en el grado de muy tóxico $\left(10<\mathrm{CL}_{50}<100 \mathrm{ug} / \mathrm{ml}\right)$. El aceite de cáscara de limón $25 \%$ no se determinó el $\mathrm{CL}_{50}$ debido a que todos los nauplios sobrevivieron. Los compuestos volátiles identificados a partir del cromatograma del aceite esencial de cáscara limón $100 \%$ fueron 39 , con mayores porcentajes en el Limoneno $(35,03 \%)$, g-Terpineno $(11,86 \%)$, Terpinoleno $(10,22 \%)$ y $\alpha$ Terpinoleno (9,63\%). Asimismo, los compuestos del aceite esencial de cáscara de naranja, se identificaron 25 compuestos, observándose el mayor porcentaje en el D-limoneno $(92,94 \%)$.

\section{DISCUSIÓN}

Se comparó la disolución de la gutapercha de uso endodóntico utilizando un aceite de cáscara de naranja comercial y de cáscara de limón, así como el ensayo de toxicidad aguda con Artemias franciscanas. La disolución de la gutapercha se determinó midiendo la diferencia de peso antes y después de exponerlos en las sustancias evaluadas, en el proceso se observó que su estructura se desintegra luego de la inmersión a los diferentes solventes por lo que este material va perdiendo peso. Los protocolos de trabajo fueron reproducidos según los estudios de investigación de Magalhaes et al.(7) Pineda et al. (8), Mushtap et al., (20), sin embargo, ellos no explican estos hallazgos, dando a entender que la disolución está dada por pérdida de masa la cual puede haber sucedido; al revisar sus métodos no especifican si los conos o discos con gutapercha están en contacto o no con alguna superficie por lo cual parte de la masa podría perderse por el reblandecimiento o desprendimiento del material al quedarse adherido a la superficie, como se observó en esta investigación. A la evaluación al estereomicroscopio se evidenció como parte de la masa externa se desprende, sobre todo con la inmersión al aceite de naranja y xilol. En este último no solo se observa una disolución, sino que además una deformación del cono, con indicios de fisuras en el cuerpo de la gutapercha, estas características no se observan luego de la inmersión al aceite de limón. El aumento de volumen probablemente este dado por la porosidad que existen en los conos de gutapercha, como lo demuestra en su estudio de Mondragon (21) quien encontró en la masa interna del cono de gutapercha, huecos homogéneos en todos sus tercios; por lo tanto, se presupone que el aumento de volumen este asociado a la incorporación del solvente en los conos de gutapercha.

No se encontró diferencia significativa en el peso de la gutapercha expuesta al aceite de cáscara de naranja con el de aceite de limón 100\% y 75\% (p>0,05), a diferencia de las demás diluciones del aceite de cáscara de limón $50 \%$ y $25 \%(\mathrm{p}<0,05)$, probablemente debido a la mayor cantidad de alcohol en la solución, este no ejerza ningún efecto sobre la gutapercha; asimismo, el tiempo no influyó en la disolución de los conos de gutapercha en las sustancias evaluadas $(p>0,05)$ (5), contrariamente a lo encontrado por Yadav et al. (22), quienes encontraron que a mayor tiempo mayor disolución de selladores endodónticos. Los solventes son necesarios, como lo demuestra Obeid y Nagy (23) donde encontraron que los solventes resinosos son eficaces para remover resina y MTA, así mismo Bayram et al. (24) determinaron que los solventes de gutapercha como el cloroformo, endosolv, aceite de naranja y eucaliptol, disminuyen la fuerza de adhesión de la biodentina y mineral trióxido agregado en forma de cápsula. Algunos estudios han demostrado la influencia de los solventes sobre la integridad de los canales radiculares, como lo investigado por Karatas et al. (25) donde determinaron que el cloroformo permitió mayor trasportación de los canales radiculares que el eucaliptol y aceite de naranja durante el retratamiento endodóntico. La disolución puede estar dada por los tipos y cantidad de compuestos de los aceites, como lo que se observa en la cromatografía de gases; donde se identificó 39 compuestos en el aceite de cáscara de limón $100 \%$, a diferencia del número de los 25 compuestos encontrados en el aceite de cáscara de naranja. El Limoneno fue el compuesto con más porcentaje encontrado en el aceite de cáscara de limón (35\%); y el D Limoneno fue el componente con más porcentaje encontrado en el aceite de cáscara de naranja (92\%), siendo estos probablemente los responsables del reblandecimiento y disolución de la gutapercha, algún grupo o el conjunto de todos los compuestos los responsables de los fenómenos.

No se encontró diferencia significativa al evaluar el xilol y el aceite de cáscara de naranja, resultados similares fueron encontrados por Pécora et al. (6), 
de igual manera Mushtap et al. (20) con metodología similar a la presente investigación, quienes utilizaron conos de gutapercha $\mathrm{N}^{\circ} 40$ (0,06 de conicidad), concluyendo que no hubo diferencia significativa en la cantidad de gutapercha disuelta a los 2 y $5 \mathrm{~min}$; a diferencia de Ramos et al. (26) que usando conos de gutarpercha Protaper F3 concluyeron que la mayor capacidad de solvencia fue obtenida con xilol, seguido del aceite de naranja , limón y eucaliptol; asimismo, Ramírez comparó los aceites de naranja y limón, encontrando que el aceite esencial de cáscara de limón fue más efectivo que el aceite esencial de cáscara de naranja $(\mathrm{p}<0,05)(27)$; a diferencia de Pineda et al., quienes concluyeron que el xilol presentó efectos solventes superiores en todos los tiempos de experimentación (8); a diferencia del presente estudio donde no se encontró diferencia significativa en todos los tiempos evaluados $(p>0,05)$. Para determinar el riesgo toxicológico existen varios ensayos tanto in vivo como in vitro, en este trabajo se utilizó un ensayo in vitro que es el ensayo toxicológico con Artemia franciscana, el cual está siendo ampliamente utilizado para este fin (14-17).

En esta investigación para evaluar la concentración letal media $\left(\mathrm{CL}_{50}\right)$ de los aceites esenciales, fueron realizados pruebas piloto sobre la Artemia franciscana, con el fin de establecer el rango de concentraciones de las diluciones que permitió la determinación de la $\mathrm{CL}_{50}$ por el método de análisis de Probit establecido por Finney (12). Para realizar los bioensayos de letalidad fueron preparadas las soluciones de 0,$1 ; 1 ; 10 ; 50 ; 100 ; 250$ y $500 \mathrm{ug} / \mathrm{mL}$ para el xilol; y 15,30 , 45, 60 y $75 \mathrm{ug} / \mathrm{ml}$ para evaluar a los aceites esenciales. Se establecieron estos rangos luego de realizar varios estudios pilotos para ajustar la concentración sobre todo para evaluar los aceites esenciales, debido que en rangos superiores a $100 \mathrm{ug} / \mathrm{mL}$ no se podía determinar el $\mathrm{LC}_{50}$. Bajo las condiciones de este estudio, todos los solventes fueron tóxicos $(\mathrm{p}>0,05)$, los aceites esenciales a excepción del aceite esencial de cáscara de limón $25 \%$, se encontraron en la categoría de toxicidad elevada $(10-100 \mathrm{ug} / \mathrm{mL})$. En el aceite esencial de cáscara de limón $25 \%$ no se encontró toxicidad debido a que en el ensayo todos los nauplios vivieron. La causa de la toxicidad de los aceites en este ensayo puede ser probablemente al efecto irritante que tienen los aceites esenciales o al cambio del $\mathrm{pH}$ en las soluciones evaluadas. El xilol presentó una toxicidad moderada por tener un $\mathrm{LC}_{50} \mathrm{de}$ $258 \mathrm{ug} / \mathrm{mL}$ (100-500 ug/mL), a diferencia de estudios realizados Nagayama (10) que utilizando fibroblastos de ligamento NIH 3T3, encontró que el xilol fue más tóxico; mientras que Zaccaro et al., (11), usando macrófagos peritoneales de ratones suizos, concluyó que el aceite de naranja mostró menor citotoxicidad que el cloroformo y el aceite de eucalipto.

Según los resultados de la presente investigación, el aceite cáscara de limón podría orientar a nuevos trabajos que puedan fortalecer sus beneficios a un posible uso para el profesional odontólogo, basado en un producto natural y peruano. Si bien es cierto que la prueba de citotoxicidad con Artemia franciscana es útil, una prueba con línea celular sería más exacta, se recomienda futuros estudios para confirmar estos resultados.

\section{CONCLUSIÓNES}

El aceite de cáscara de limón $100 \%$ y $75 \%$ fue tan efectivo para disolver la gutapercha como el aceite de cáscara de naranja y xilol. Todos los aceites fueron tóxicos, a excepción del aceite esencial de cáscara de limón 25\%. El limoneno y D limoneno fueron los componentes con mayor cantidad en el aceite de cáscara de limón y de cáscara de naranja, respectivamente.

\section{Correspondencia}

Paul M. Herrera

Correo electrónico: pherrera@ucv.edu.pe

\section{REFERENCIAS BIBLIOGRAFICAS}

1. Lima M. Endodoncia de la Biología a la Técnica. Sao Paulo: Amolca; 2009.

2. Cohen S, Burns R. Vías de la pulpa. Madrid: Elsevier; 2007.

3. Ingle J, Bakland L. Endodontics. Ciudad de éxico: Mc Graw Hill Interamericana; 1996.

4. Portales A, Mendoza O. Estrategias para la competitividad sostenible del limón en Piura. Lima: PUCP-CENTRUM; 2007.

5. Pécora J, Da Costa W, Filho D, José S. Apresentação de um óleo esencial obtido ce citrus arantium, eficaz desisntegração do cimento de óxido de zinco-eugenol do interior do canal radicular. Odonto. 1992; 1(5):130-2.

6. Pécora J, Spanó J, Barbin E. Estudo "in vitro" sobre o amolecimiento de cones de guta-perca no re-tratamiento 
endodóntico. Braz Den J. 1993; 4(1):43-7.

7. Magalhaes B, Johan J, Lund R, Martos J, Del Pino. Dissolving efficacy of some organic solvents on guttapercha. Braz Oral Res. 2007; 21(4):303-7.

8. Pineda M, Palacios E. Evaluación in vitro de tres solventes de gutapercha. Odontol. Sanmarquina. 2011; 14(1): 15-18.

9. De Lima C, Da Silva C, De Oliveira Costa D, Scienza M, Silva M, Da Silva R. Caracterização química do óleo essencial da casca do citrus sinensis obtido por hidrodestilação em Aparelho Clevenger. Belem-Pa: Universidad Federal Do Pará Instituto de Tecnologia. Facultad de Engenharia química; 2010.

10. Nagayama K. Avaliacäo comparativa da citotoxicidade in vitro dos solventes utilizados no retratamiento endodôntico. Dissetacäo para obter o Título de Mestre. Sao Paulo: Universidade de Sao Paulo;2003.

11. Zaccaro M, Oliveira R, Blaudt F, Faria S. In vitro evaluation of macrophage viability after incubation in orange oil, eucalyptol, and chloroform. Oral Surg Oral Med Oral Pathol Oral Radiol Endod. 2006; 102:24-7.

12. Fernandez-Calienes A, Mendiola J. Evaluación de la toxicidad de extractos de plantas cubanas con posible acción antiparasitaria utilizando larvas de Artemia salina L. Rev Cubana Med Trop. 2009; 61(3):254-8.

13. Michael AS, Thompson CG, Abramovitz M. Artemia salina as a test organism for bioassay. Science. 1956;123(3194):464.

14. Lima L, Barreto L, Lopes R, et al. Evaluation of the larvicidal potential of the essential oil pogostemon cablin (Blanco) Benth in the control of Aedes aegypti. Pharmaceuticals (Basel). 2019;12(2): 53.

15. Mashjoor S, Yousefzadi M, Zolgharnein H, Kamrani E, Alishahi M. Phyco-linked vs chemogenic magnetite nanoparticles: Route selectivity in nano-synthesis, antibacterial and acute zooplanktonic responses. Mater Sci Eng C Mater Biol Appl. 2019;102: 324-40.

16. An HJ, Sarkheil M, Park HS, Yu IJ, Johari SA. Comparative toxicity of silver nanoparticles (AgNPs) and silver nanowires (AgNWs) on saltwater microcrustacean, Artemia salina. Comp Biochem Physiol C Toxicol Pharmacol. 2019; 218: 62-9.

17. Kachenton S, Jiraungkoorskul W, Kangwanrangsan N, Tansatit T. Cytotoxicity and histopathological analysis of titanium nanoparticles via Artemia salina. Environ Sci Pollut Res Int. 2019;26(15):14706-11.
18. Lagarto A, Silva R, Guerra I, Iglesias L. Comparative study of the assay of Artemia salina L. and the estimate of the medium lethal dose (LD50 value) in mice, to determine oral acute toxicity of plant extracts. Phytomedicine. 2001; 8(5):395-400.

19. Ruiz-González LE, Vázquez-Zea JA, Vega-Villasante F, Guzmán-Dávalos L, Guerrero-Galván SR. Evaluation of the toxicity of Basidiomycota fungi on the hatching of Artemia franciscana cysts. Rev Iberoam Micol. 2017;34(4):220-4.

20. Mushtap M, Farooq R, Ibrahim M, Yaqoob F. Dissolving efficacy of different organic solvents on guttapercha and resilon root canal obturating materials at different immersion time intervals. J Conserv Dent. 2012; (15):2:141-5.

21. Mondragón M. Estudio descriptivo de la gutapercha PRODENT por medio de MEB y EDX in vitro. Revista A D M. 2002;59(6):211-5.

22. Yadav HK, Yadav RK, Chandra A, Thakkar RR. The effectiveness of eucalyptus oil, orange oil, and xylene in dissolving different endodontic sealers. J Conserv Dent; 2016;19(4):332-7.

23. Obeid M.F. Retreatability of different endodontic sealers using chemical solvents. Tanta Dental Journal Journal. 2015;12(4):286-291.

24. Bayram E, Bayram HM, Aslam T, Göktürk H, Ustün Y. Evaluation of calcium silicate cement bond strength after using gutta-percha solvents. Nijer J Clin Pract 2017;20:1417-21.

25. Karatas E, Kol E, Bayrakdar I, Arslan H. The effect of chloroform, orange oil and eucalyptol on root canal transportation in endodontic retreatment. Aut Endod J. 2016; 42: 37-40.

26. Ramos TI, Câmara AC, Aguiar CM. Evaluation of capacity of essential oils in dissolving protaper universal gutta-percha points. Acta Stomatol Croat. 2016;50(2):128-33.

27. Ramírez M. Comparación in vitro del aceite esencial de cáscara de limón y el aceite esencial de cáscara de naranja como solventes de gutapercha de uso endodóntico. Tesis de maestría. Trujillo: Universidad Nacional de Trujillo; 2017

Recibido: 23-01-2019

Aceptado: 07-06-2019 